\title{
Influence of color and luminance on the Müller-Lyer illusion
}

\author{
KAREL J. SADZA and CHARLES M. M. de WEERT \\ University of Nijmegen, Nijmegen, The Netherlands
}

\begin{abstract}
The arrowheads and lines of the Müller-Lyer illusion were varied independently in color and luminance. Stimuli were presented on a computer-controlled color television monitor. Subjects responded yes or no to a forced choice. Results indicated the following: (1) Differences in color between arrowheads and line reduce the illusion. (2) Luminance differences between arrowheads and line always reduce the illusion. (3) Effect 1 and 2 are independent of each other. (4) With a dark background, if the arrowheads are brighter than the line, the decrease is smaller than when the arrowheads are darker than the line. (5) A total change of luminance does not affect the illusion. Results 1 and 2 contradict Wickelgren's contrast hypothesis, which predicts a maximum illusion with the difference between arrowheads and background at maximum and the difference between line and background at minimum. Result 4, however, shows that an explanation in terms of similarity only is not possible. Therefore, a description in terms of both contrast and similarity is proposed.
\end{abstract}

In 18́89, Müller-Lyer reported that the size of a line that is delimited by two pairs of in-going oblique lines is underestimated and that the size of a line that is delimited by two outgoing oblique lines is overestimated (Figure 1). Experimental results indicate that there are systematic relations between certain aspects of the Müller-Lyer figure and it's perceived size. For instance, experiments in which the geometrical properties of the figure were varied have led to good insight in to the effects of variation of angle and length of the oblique lines (Davies \& Spencer, 1977; Restle \& Decker, 1977).

Two other factors that have an influence on the Müller-Lyer illusion are color and luminance of the figure in relation to the background. Researchers interested in the influence of color and brightness on the Müller-Lyer illusion can be divided in two groups. One group used a Müller-Lyer figure in which the line and the arrows had the same color and luminance. The second group used lines and arrows of different colors.

The researchers in the first group, who concentrated on the effect of brightness, were Gregory (1977), Pollack (1970b), Ebert and Pollack (1972b), and Butler (1981). Gregory (1977) used stimuli with a color different from that of the background. He varied the luminance of the figure in relation to that of the background and was especially interested in the size of the illusion when figure and ground were equally bright, that is, isoluminant. According to his obser-

K. J. Sadza's current address is: Institute for Perception TNO, P. O. Box 23, 3769ZG Soesterberg, The Netherlands. Ch. M. M. de Weert's mailing address is: University of Nijmegen, Department of Experimental Psychology, P. O. Box 9104, 6500HE Nijmegen, The Netherlands. vations, this case gave no noticeable change in the size of the illusion. Pollack and Silvar (1967) however, found that the extent of the illusion was greatest for people with less pigmentation of their fundus oculi. They based this conclusion on the fact that a given contrast is perceived less when this pigmentation is stronger. The contrast effect, as such, would be caused by lateral inhibition. Butler (1981) found no effects of brightness contrast between figure and ground.

Pollack (1970a) and Ebert and Pollack (1972a) also found effects of color when figure and background had the same luminance. Among other things, Pollack found that, with the same gray background, a red figure caused a greater illusion than a green one.

The first experiment in which arrows and line were not the same was one performed by Bates (1923). She found that, with a black line and a white background, black arrows caused a greater illusion than did gray ones. She interpreted this as a sameness effect: the greater the difference between line and arrow, the weaker the illusion.

Mukerji (1957) conducted a similar experiment for color. He found that, with a black line, black arrows caused a larger illusion than did red or green ones. $\mathrm{He}$, too, explained his results in terms of sameness.

Wickelgren (1965) questioned the sameness hypothesis because neither Bates nor Mukerji checked on luminance. She used a model in which luminance contrast between arrows and background in relation to the luminance contrast between line and background determines the strength of the illusion. She called this ratio the "oblique effectiveness ratio." Her contrast hypothesis predicts that the illusion will not be maximal when line and arrows are equal to 
each other, but will be when the luminance contrast between arrows and background is maximal and the luminance contrast between line and background is minimal. Her results point in this direction. Wickelgren did not take the influence of color into account.

Weintraub, Tong, \& Smith (1973) confirmed Wickelgren's findings, and Oyama (1962) found similar results for the Delboeuf illusion. Here brightness was the only determining factor and neither color nor sameness had any effect.

In the experiments to be described below, color and luminance of line and arrows were varied quantitatively.

\section{EXPERIMENT 1: EFFECT OF COLOR DIFFERENCES AT EQUAL LUMINANCE}

The first experiment was primarily meant to find out whether Wickelgren's contrast hypothesis also held for colored stimuli. If only the luminance ratio between arrows and line determines the size of the illusion, differences in color should not have any effect, provided the luminances are equal.

A second point concerns the role of retinal blur. When arrows and line are of a different color, there is, due to chromatic aberration, more retinal blur. This blur could lead to a larger illusion (Coren, Ward, Porac, \& Fraser, 1978; Eijkman, Jongsma, \& Vincent, 1981; Zucker, 1980) and a greater variability.

\section{Method}

Subjects. In this experiment, 15 subjects took part. For most of them, participation was a condition for obtaining their degrees in psychology. All subjects had normal color vision abilities according to the Ishihara test.

Stimuli. The stimuli consisted of a horizontal line, divided into two parts, with three arrowheads (Figure 1). The length of the outgoing part was $6.5 \mathrm{~cm}$ (visual angle: $1 \mathrm{deg} 33 \mathrm{~min}$ ), but the length of the ingoing part was variable. The length of the oblique lines was $1.6 \mathrm{~cm}(23 \mathrm{~min})$. The angle between horizontal and oblique was $37 \mathrm{deg}$. The thickness of the lines was $1 \mathrm{~mm}(1 \mathrm{~min}$ $26 \mathrm{sec}$ ) (two lines of the television screen). The luminance of all the colors was $40 \mathrm{~cd} / \mathrm{m}^{2}$, measured with a Pritchard photometer with a photopic filter. The background was a rectangular plane with a luminance of $1 \mathrm{~cd} / \mathrm{m}^{2}$. The size of this plane was $34 * 25 \mathrm{~cm}$ (8* 6 deg). Red, yellow, green, and white were used as stimulus colors. The coordinates of the colors in the color triangle were: red $(0.654,0.341)$, yellow $(0.485,0.463)$, green $(0.285,0.606)$ and white $(0.282,0.247)$. All 16 color combinations of line and arrows were presented to the subjects.

Apparatus. A Barco R,G,B videomonitor, controlled by an LSI/23 microcomputer, was used as the stimulus generator. A detailed description of this apparatus can be found in Wittebrood, Wansink, and De Weert (1981).

Procedure. Each session consisted of 16 blocks each of 32 trials.' Within each block, all 16 combinations of color for line and arrows were presented twice in a random sequence. The subjects were seated in an almost dark room at a distance of $2.40 \mathrm{~m}$ from the video monitor. Viewing time was chosen such that a compromise was obtained between a comfortable viewing time, allowing for several fixations, and a reasonably short total experimental session time. We took $1.5 \mathrm{sec}$ as the presentation time. After this, the stimulus disappeared and the subject could indicate, by pressing one of two buttons, which half of the stimulus appeared to be longer. He or she was asked to press the left button if the left

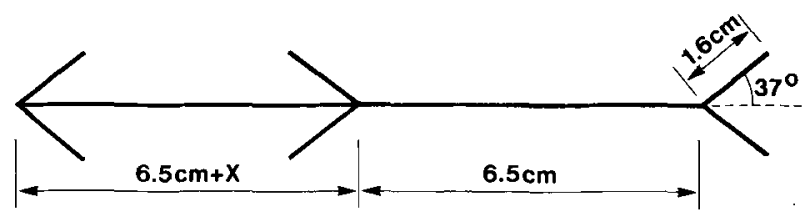

Figure 1. Stimulus used. In this article, the left half is called the "ingoing part" and the right half is called the "outgoing part."

half was the longer and the right button if the right half was the longer. The ingoing part of the Muiller-Lyer figure was presented left or right at random. In order to keep the subject from basing his judgment on the position of the figure in relation to the background, the position of the figure was varied. With each presentation, it was moved maximally $13 \mathrm{~mm}$ in horizontal direction and $9 \mathrm{~mm}$ in vertical direction with respect to the previous presentation.

After the response of the subject, there was a pause of $1 \mathrm{sec}$ before the next stimulus appeared. The whole session was divided into four series of 128 trials each, one series lasting from 8 to $9 \mathrm{~min}$. Between each series, the subjects performed a simple brightnessjudgment task, lasting about $3 \mathrm{~min}$. This was done to break the monotony of the experiment a little.

In order to find the length at which the subject perceived both halves of the figure to be equally long (the PSE: point of subjective equality), an up-and-down method was used. The subject was shown a Muller-Lyer figure of which the ingoing part was a certain percentage longer than the outgoing part. If the subject thought the ingoing part was shorter than the outgoing part, the same color/luminance combination was shown in the next block with the ingoing part a little $(6 \%$ or $12 \%)$ longer. If the subject perceived the ingoing part to be longer than the outgoing part, the ingoing part of the stimulus in the next block would be a little shorter $(6 \%$ or $12 \%)$. There were 32 presentations in all for each of the 16 different stimuli. These 32 presentations were divided over two staircases. One staircase became $6 \%$ shorter after a "toolong" response and $12 \%$ longer after a "too short" response. The other staircase became $12 \%$ shorter and $6 \%$ longer. The interval size of $6 \%$ was chosen because this corresponded with the standard deviation per subject found in pilot experiments. According to Dixon (1965), an interval size between .67 and 1.50 gives the best estimate of the PSE. The mean accuracy with which subjects in the following experiments judged the stimuli was $0=7.8 \%$. In order to be in a better position to calculate this accuracy later, two asymmetrically reacting staircases were used. These staircases diverge if the subject responds indifferently and are stable around the points at which $67 \%$ of the answers are "too short" or "too long." In the analysis, the responses of the two staircases were joined, as can be seen in Figure 2.

Anglysis. Through the response percentages, a cumulative Gauss curve was fitted by means of probit analysis after a maximum likelihood method (Finney, 1971). The 50\% point of this curve counted as PSE (Figure $2 \mathrm{~b}$ ). From the angle between this curve and the horizontal at the $50 \%$ point, the standard deviation may be calculated. This was used to measure the accuracy with which the subject performed his task.

Sometimes, for only one size, the response frequency was not equal to $0 \%$ or $100 \%$. In such cases, application of probit analysis was impossible and the PSE was determined by linear interpolation.

The PSEs that were found by this method were used as input for an analysis of variance according to a randomized block design with subjects as block. The accuracies, too, were analyzed by analysis of variance according to the same design.

\section{Results}

The results are shown in Figure 3. Analysis of variance showed a significant effect for combination 

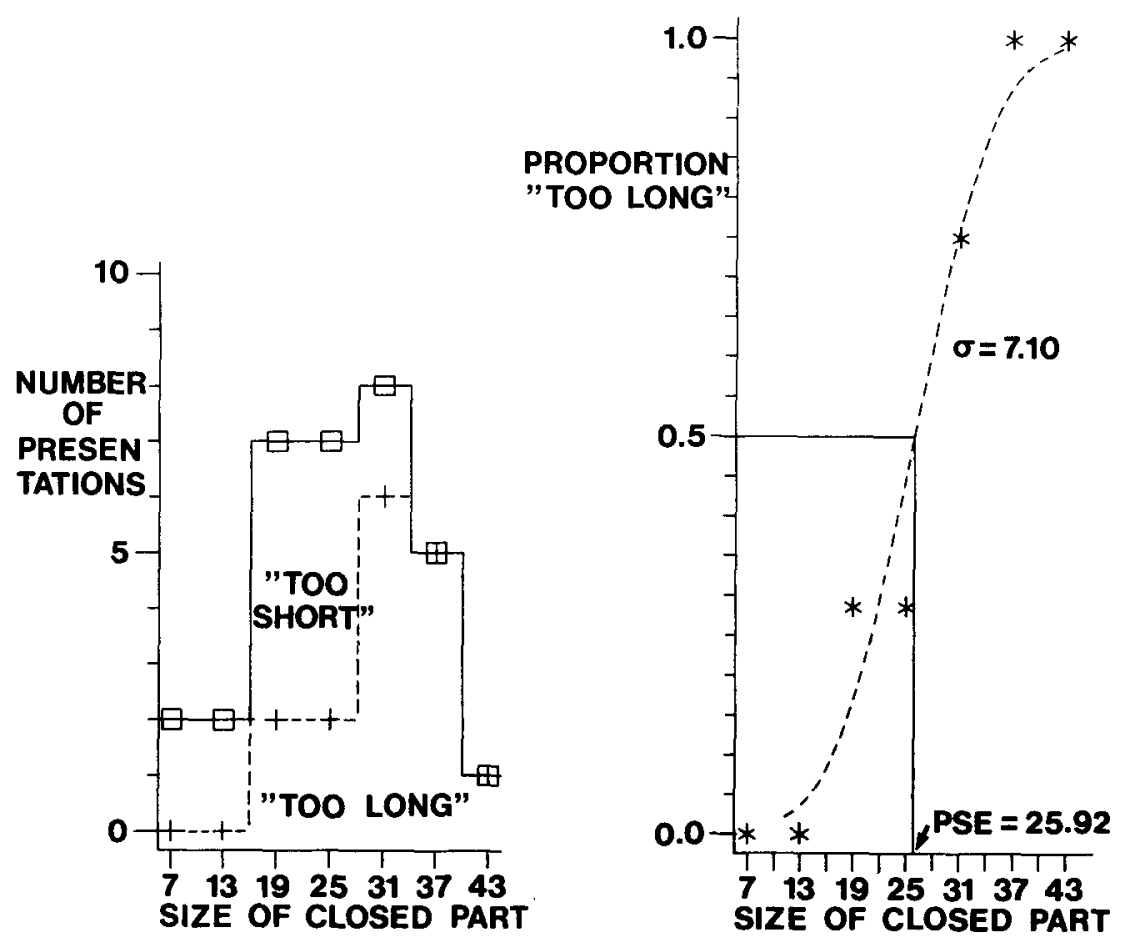

Figare 2. Example of determination of PSE and aceuracy.

$[F(15,210)=12.08, p<.0001]$. From Figure 3, it is also clear that one-color figures create a larger illusion than do figures with two colors. On the right in Figure 3, the means are shown for one-colored and two-colored stimuli as a group. The differences between one-colored and two-colored stimuli is signifi-

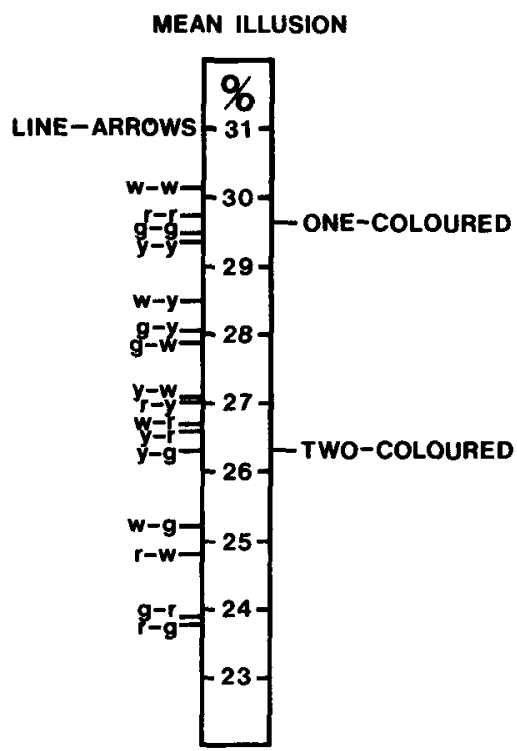

Figure 3. Ilusion decrement due to color differences at equal luminance. Left: Mean illusion per color combination. Right: Mean illusion for one and two colored stimull. cant $[F(1,224)=75.05, p<.0001]$. Analysis within the group of one-colored stimuli showed no significant effects.

The mean accuracy figure for the subjects was $8.1 \%$. Analysis of the accuracies showed no differences between color combinations $[F(15,208)=0.88$, p > .59]. The subjects, however, did differ significantly $[F(14,208)=8.08, p<.0001]$. The mean accuracies per subject were between $4.5 \%$ and $11 \%$. The subjects also differed strongly in perceived size of the illusion $[F(14,210)=156.24, p<.001]$. The mean illusions per subject were between $15 \%$ and $36 \%$.

\section{Discussion}

It is clear that color differences play a role in the size of the Muller-Lyer illusion. In any case, it can be said that as long as luminances are equal, the greatest illusions are evoked by figures in which the arrows are most similar to the line. This conflicts with Wickelgren's contrast hypothesis and suggests a sameness explanation. In Experiment 4, we will show that this result cannot be explained by possible luminance differences between the colors.

The fact that no differences in accuracy between conditions were found shows that the Muller-Lyer illusion cannot be seen as the imprecise perception of the position of intersecting lines, because in that case the inaccuracy should increase together with the PSE. Also, color effects caused by chromatic aberration would make us expect changes in accuracy. 
The differences found between subjects show that it is necessary to compare conditions within subjects wherever possible.

\section{EXPERIMENT 2: EFFECTS OF BRIGHTNESS CONTRAST}

The results with colored stimuli do not confirm predictions based on the ideas of Wickelgren (1965). For that reason, and in order to see to what extent Wickelgren's results could be replicated with our method, it seemed justified to do an experiment in which only luminance was varied.

\footnotetext{
Method

Subjects. Again, 15 students took part as a course requirement. Stimuli. The stimuli were the same as in Experiment 1. The color of line and arrows was always white, but there were four different luminances: $40,20,10$, and $5 \mathrm{~cd} / \mathrm{m}^{2}$. The stimuli were presented in all possible luminance combinations. The luminance of the background was always $1 \mathrm{~cd} / \mathrm{m}^{2}$.

Procedure. The procedure was the same as in Experiment 1.
}

\section{Results}

In Figure 4, the results are presented graphically as a function of luminance ratio. As can be seen, an increase in luminance ratio between arrows and line always leads to a decrease in illusion. This decrease is significant $[F(6,219)=27.38, p<.0001]$. This effect is stronger when the arrows have a lower contrast with the background than does the line. Striking in Figure 4 is the fact that there is no noticeable difference between positive and negative contrast of a factor two. Analysis of the subgroup of stimuli in which line and arrows had equal luminance did not show a general brightness effect $[F(3,42)=0.05$, $p>$.98]. The mean accuracy was $8.0 \%$. Analysis of the accuracies showed no significant differences between conditions $[F(6,214)=0.98, p>.44]$.

\section{Discussion}

The absence of a general brightness effect agrees with Butler's (1981) findings. Ebert and Pollack (1973), however, did find contrast effects for this condition. They also found that contrast interacted with viewing time. An important difference between their experiment and both Butler's and ours is that Butler and we used transmitted light instead of reflected light. This enabled us to use much higher light intensities. It is possible that above a certain contrast ratio between figure and ground, larger luminance differences have no more effect. Maybe these high ratios also account for the absence of an effect when the inducing lines are twice or half as luminant as the test lines. Although Ebert and Pollack (1973) found more clearly different contrast effects for $1,500 \mathrm{msec}$ than for other durations, it seems unlikely to us that the absence of a contrast effect in our data is due to an accidental choice of viewing time. Butler, whose luminances were also very high, did not find a con-

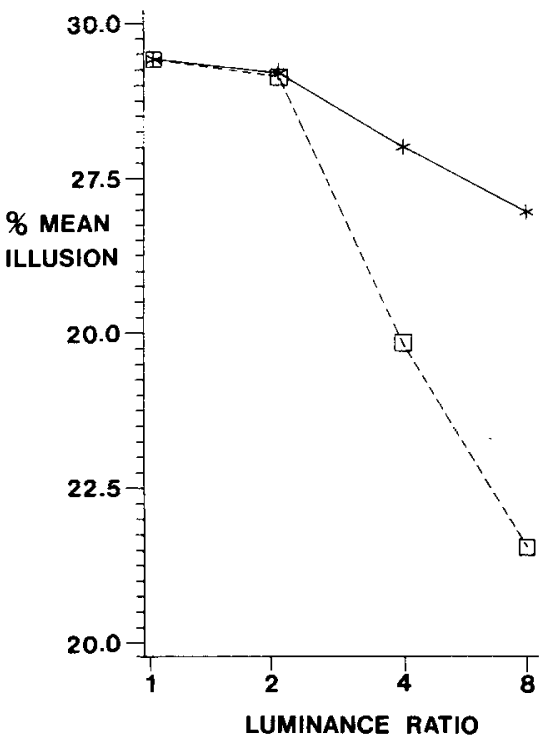

Figure 4. Decrease of the illusion due to luminance differences between arrows and line. Continuous line: Arrows brighter than line. Interrupted line: Arrows darker than line. The color of the stimuli was always white.

trast effect for a much longer viewing time. Ebert and Pollack (1973), using lower luminances, found contrast effects for all tested viewing times between 500 and $18,000 \mathrm{msec}$.

The decrease in illusion when luminance contrast for the arrows decreases agrees with Bates (1923) and Wickelgren (1965). The fact that the illusion also decreases when luminance contrast for the arrows increases, however, is contrary to the results of Wickelgren (1965).

\section{EXPERIMENT 3: INCREASE OF CONTRAST OF THE ARROWS WITH ONE-COLORED STIMULI}

The effect of increase of luminance contrast, as found in Experiment 2, conflicts with the known literature. Therefore, it seemed sensible to try to replicate it. At the same time, we wanted to know whether this effect could also be found for hues other than white.

\section{Method}

Subjects. Eighteen subjects took part. The results of one subject were left out of the analysis because of unacceptable inaccuracies.

Stimull. The stimuli were presented in red, yellow, green, and white. The luminance ratios between the arrows and the line were $1\left(20: 20 \mathrm{~cd} / \mathrm{m}^{2}\right), 2\left(20: 10 \mathrm{~cd} / \mathrm{m}^{2}\right), 4\left(40: 10 \mathrm{~cd} / \mathrm{m}^{2}\right)$, and $8(40: 5$ $\left.\mathrm{cd} / \mathrm{m}^{2}\right)$.

Analysis. The PSEs were analyzed according to a complete randomized block design, with subject as block and color and luminance ratio as factors.

\section{Results}

The results are given in Figure 5. It confirms the earlier finding that an increase in luminance ratio 
causes a decrease in illusion. Both luminance ratio $[F(3,249)=11.32, p<.0001]$ and color $[F(3,249)=$ $5.53, \mathrm{p}<.0012]$ are significant. The mean accuracy of the subjects was $7.1 \%$. Neither luminance ratio $[F(3,238)=1.00, p>.39]$ nor color $[F(3,238)=0.34$, $p>.80]$ had a significant influence on accuracy.

\section{Discussion}

It appears that the decrease found in the experiment can be generalized to several colors, at least as long as arrows and line are of the same color. The effects of color (red $>$ yellow $>$ green) are the same as those Pollack (1970a) found under isoluminant conditions.

\section{EXPERIMENT 4: COMBINED EFFECTS OF COLOR AND LUMINANCE}

In this experiment, we wanted to investigate to what extent effects of color and luminance were independent of each other. In view of the duration of the experiments, the group of subjects was divided into two groups. For the first group, the luminance ratios were 1 and 2; for the second group, they were 2 and 4.

\section{Method}

Subjects. There were 15 subjects in the first group and 20 in the second. With the second group, the results of one subject were not included in the analysis because of unacceptable inaccuracies.

Stimul. The colors of arrows and line were red and green in every possible combination. Red-green was chosen because, in Experiment 1, this combination showed the largest fall in illusion. In the first part of the experiment (i.e., for the first group of sub-

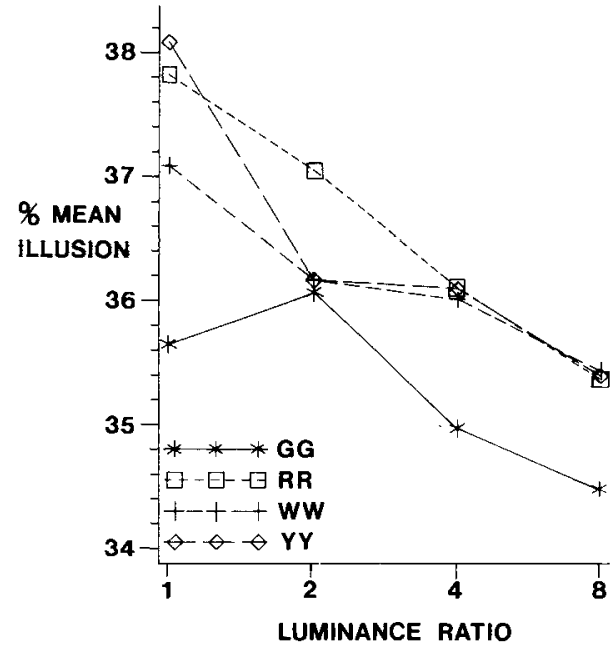

Figure 5. Decrease of the illusion due to increasing luminance ratio between arrows and line. Arrows are always brighter than the line. Line and arrows have the same color.

jects), the luminances for the arrows and line were 40 and $20 \mathrm{~cd} / \mathrm{m}^{2}$ in every possible combination. In the second part of the experiment, the luminances were 40 and $20 \mathrm{~cd} / \mathrm{m}^{2}$ for luminance ratio 2 and 40 and $10 \mathrm{~cd} / \mathrm{m}^{2}$ for luminance ratio 4 . The background was always $1 \mathrm{~cd} / \mathrm{m}^{2}$.

Analysis. The two parts of the experiment were analyzed separately according to a complete randomized block design, with subject as block and color combination and luminance ratio as factors.

\section{Results}

In Figure 6, the results are shown graphically as so-called least squares means, which are, because of
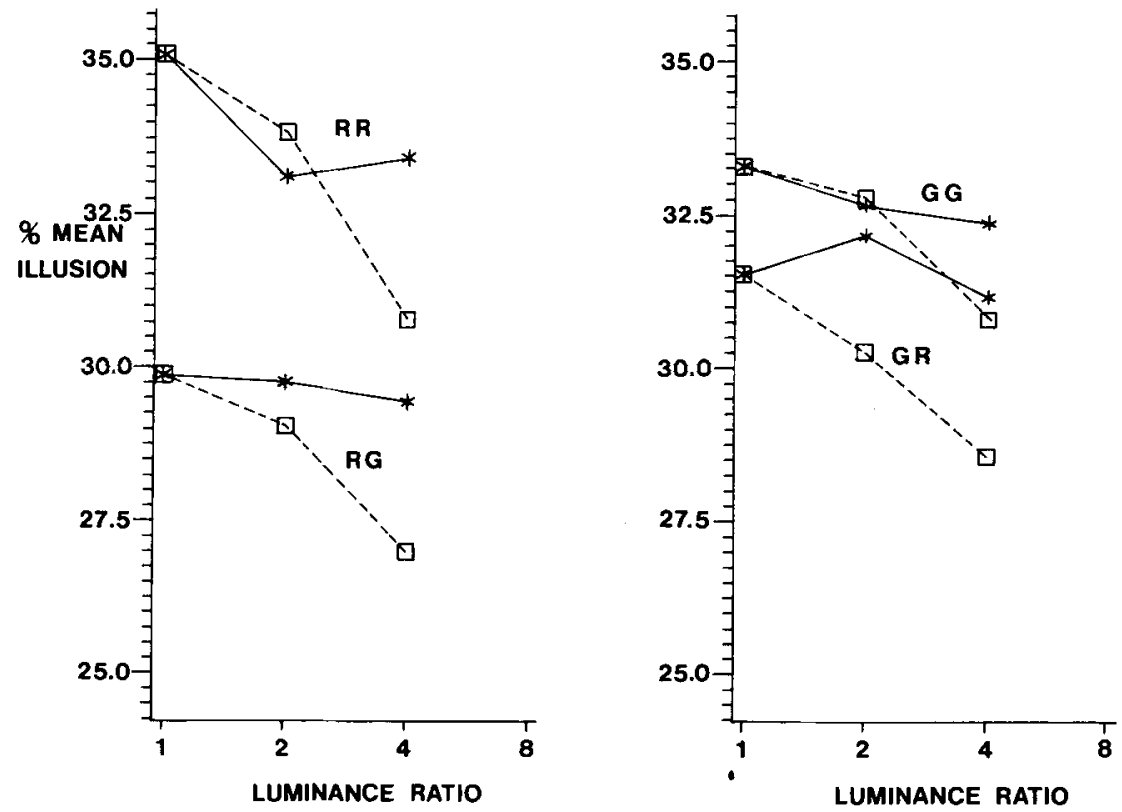

Figure 6. Least square mean for combinations of color and laminance. Conthuous line, arrows brighter; interrupted line, arrows darker. 
unequal cell sizes, corrected for individual subject means.

In the first part (luminance ratio 1 and 2), color combination was significant $[\mathrm{F}(3,220)=35.47, \mathrm{p}<$ $.0001]$ but luminance ratio was not. In the second part, both color combination $[F(3,279)=37.28$, $\mathrm{p}<.0001]$ and luminance ratio $[F(3,279)=18.62$, $\mathrm{p}<.0001]$ were significant. The order of the color effects was the same for both parts (RR > GG > GR > RG).

Analysis of the subgroup of stimuli, for which arrows and line had the same luminances, showed no significant effect for luminance $[F(1,101)=0.09$, $p>$.77]. This finding agrees with Butler's (1981).

The mean accuracy with which subjects judged the stimuli was $8.0 \%$. Between conditions, there were no significant differences in accuracy.

\section{DISCUSSION AND CONCLUSION}

Again, a color difference causes a clear decrease in illusion. The differences between one-colored and two-colored stimuli cannot be explained by possible luminance differences between colors, due to inaccurate calibration: one-colored figures with luminance ratio 2 always cause a greater illusion than do twocolored figures without luminance differences.

The luminance effects of all four curves are roughly the same. A luminance ratio of 2 does not cause a clear effect. This agrees with Experiment 2. At a

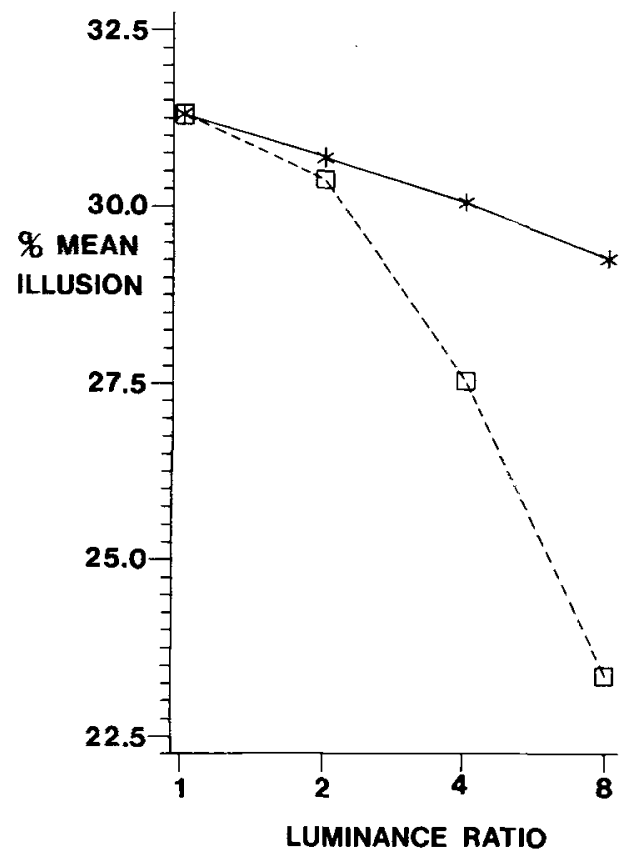

Figure 7. Least squares means for the laminance effect for all subjects.

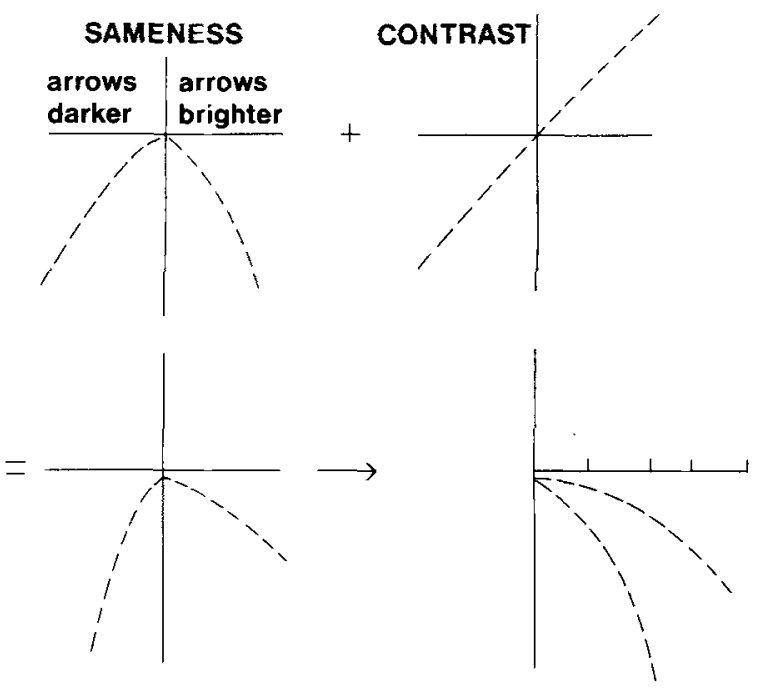

Figure 8. Explanation of the curve in Figure 7 in terms of sameness and contrast.

factor of 4 , however, the effect of luminance difference is clearly visible.

The finding that luminance differences have the same effect for two-colored and one-colored stimuli suggests that the action of color is independent from that of luminance. Figure 7 gives a curve for the effect of luminance differences. It consists of the least squares means of all 81 subjects. By now it has become clear that a contrast explanation in terms of an oblique effectiveness ratio (Wickelgren, 1965) cannot explain these data. Otherwise, the upper branch of the curve should go upward. However, a sameness explanation is not sufficient either. A dark arrow differs just as much from a light line as a light arrow does from a dark one, which means that both curves should coincide.

It is possible, however, to give a full description of the data by using a simple, additive combination of both effects. In this description, the illusion increases monotonically with the difference in luminance between arrowheads and line, whereas it decreases symmetrically with the difference in both color and luminance between these parts. Because, when dividing one curve into two, a unique solution cannot be obtained, Figure 8 provides a schematic picture of this.

\section{REFERENCES}

BAtes, M. (1923). A study of the Miller-Lyer illusion with special reference to paradoxical movement and the effect of attitude. American Journal of Psychology, 34, 46-72.

ButLen, D. B. (1981). Does brightness contrast really affect the Miuller-Lyer illusion? Perceptual and Motor Skills, 52, 623-629.

Coren, S., Ward, L. M., Porac, C., \& Fraser, R. (1978). The effect of optical blur on visual-geometric illusions. Bulletin of the Psychonomic Society, 11, 390-392. 
Davies, T. N., \& Spencer, J. (1977). An explanation for the Muiller-Lyer illusion. Perceptual and Motor skills, 45, 219-224.

Dixon, W. J. (1965). The up-and-down method for small samples. Journal of the American Statistical Association, 60, 967-978.

Ebert, P. C., \& Pollack, R. H. (1972a). Magnitude of the Muiller-Lyer illusion as a function of hue, saturation, and fundus pigmentation. Psychonomic Science, 26, 225-226.

Ebert, P. C., \& Pollack, R. H. (1972b). Magnitude of the Müller-Lyer illusion as a function of lightness contrast, viewing time, and fundus pigmentation. Psychonomic Science, 26, 347-348.

Ebert, P. C., \& Pollack, R. H. (1973). Some factors affecting magnitude of the Miller-Lyer illusion. Perceptual \& Motor Skills, 37, 433-434.

Eijkman, E. G. J., Jongsma, H. J., \& Vincent, J. (1981). Twodimensional filtering, oriented line detectors, and figural aspects as determinants of visual illusions. Perception \& Psychophysics, 29, 352-358.

Finney, D. J. (1971). Probit analysis. Cambridge University Press.

GrEGORY, R. C. (1977). Vision and isoluminant colour contrast: 1. A projection technique and observations. Perception, 6, 113-119.

Mukeru, N. (1957). Is "illusion" illusion? Journal of General Psych̆ology, 57, 209-212.

MülLER-LYER, F. C. (1889). Optische Urtheilstäuschungen. Archiv fur Anatomie und Physiologie, Physiologische Abteilung, 2, 263-270. (For a translation, see Day, R. H., \& Knuth, H. (1981). The contributions of F. C. Müller-Lyer. Perception, 10, 126-146.)

Oyama, T. (1962). The effect of hue and brightness on the sizeillusion of concentric circles. American Journal of Psychology, 75, 45-55.
Pollack, R. H. (1970a). Magnitude of the Miiller-Lyer illusion as a function of hue in the absence of lightness contrast. Proceedings of the American Psychological Association, 78, 53-54.

Pollack, R. H. (1970b). Muiller-Lyer illusion: Effect of age, lightness contrast, and hue. Science, 170, 93-95.

Pollack, R. H., \& Silvar, S. (1967). Magnitude of the MuillerLyer illusion in children as a function of pigmentation of the fundus oculi. Psychonomic Science, 8, 83-84.

Restle, F., \& Decken, J. (1977). Size of the Muiller-Lyer illusion as a function of its dimensions: Theory and data. Perception \& Psychophysics, 21, 489-503.

Weintraub, D. J., Tong, L., \& Smith, A. J. (1973). MüllerLyer versus size/reflectance-contrast illusion: Is the age-related decrement caused by a declining sensitivity to brightness contours? Developmental Psychology, 9, 6-15.

Wickelgren, B. G. (1965). Brightness contrast and length perception in the Muiller-Lyer illusion. Vision Research, 5, 141-150.

Wittebrood, J. E. M., Wansink, M. G., \& DE Weert, Ch. M. M. (1981). A versatile colour stimulus generator. Perception, 10, 63-69.

ZUCKER, S. W. (1980). The computational/representational paradigm as normal science: Further support. Behavioral and Brain Sciences, 3, 406-407.

\section{NOTE}

1. The first eight subjects were shown 25 blocks. Because these turned out to be too much to be presented within $1 \mathrm{~h}$, the number of blocks was reduced to 16 for the other subjects.

(Manuscript received June 6, 1983; revision accepted for publication December 16,1983 .) 